\title{
Evolution of the human brain
}

\author{
A human-specific gene is a determinant of the cognitive architecture of the human cerebral \\ cortex
}

\author{
Colette Dehay ${ }^{1}$ and Henry Kennedy ${ }^{1,2}$
}

\begin{abstract}
${ }^{1}$ University of Lyon, Université Claude Bernard Lyon 1, Inserm, Stem Cell and Brain Research Institute U1208, 69500 Bron, France.

${ }^{2}$ Institute of Neuroscience, State Key Laboratory of Neuroscience, Chinese Academy of Sciences Key Laboratory of Primate Neurobiology, Shanghai 200031, China.
\end{abstract}

Email: colette.dehay@inserm.fr; henry.kennedy@inserm.fr

Since early hominids emerged 5 million years ago, humans have evolved sizeable brains to support higher cognitive functions. In particular, the human cerebral cortex is greatly expanded, allowing accommodation of the evolutionary increases in the number of cortical areas, the functional modules that subserve perception, attention, motor control, cognition, memory and learning. Duplicated genes specific to the Homo lineage have played key roles in human speciation, particularly in the development of the highly complex human brain (l) and the circuits of the cerebral cortex (2). On page XXX of this issue, Heide et al. (3) identify $A R H G A P 11 B$ [Rho guanosine triphosphatase (GTPase) activating protein 11B], a human-specific duplicated gene, as a regulator of human cerebral cortex development. By expressing $A R H G A P 11 B$ in marmosets, a smooth brained primate, this study explores the influence of the gene on expanding the primate cortex.

The human neocortex is marked by an important increase in surface area and its radial dimension, the latter due to the selective enlargement of the supragranular layers (4). Supragranular neurons have an important role in the integration of ascending and descending cortico-cortical pathways that underlie information transfer and processing between the numerous hierarchically organized cortical areas in primates. Therefore, the specific expansion of the supragranular neurons contributes to the cognitive functions of primates, culminating in humans (4). Much of the origin of this expansion can be attributed to primatespecific features of corticogenesis, including an expanded progenitor pool in the developing primate cerebral cortex: the outer subventricular zone (OSVZ) (5), which includes specialized progenitors called basal radial glial cells (bRGs) (6). bRGs are endowed with extensive proliferative capacities and generate mostly supragranular neurons (7).

$A R H G A P 11 B$ has received much attention because it is specifically enriched in cortical bRGs $(8)$. When locally overexpressed in mouse or ferret cortex, ARHGAPI1B boosts bRG proliferation and increases the numbers of cortical neurons $(8,9)$. These observations suggest that this gene could link specific aspects of primate corticogenesis and characteristic features of the adult primate cortex architecture. To test this, Heide et al. expressed $A R H G A P I 1 B$ in the developing cortex of the embryonic marmoset. When $A R H G A P 11 B$ is expressed under the control of the human promoter and upstream regulatory sequences, the transgenic mid-gestation marmoset exhibits an enlarged developing cortex with signs of folding. The crucial observation is that there is a selective increase in the numbers of neurons in the supragranular layers. This "humanization" of the marmoset fetal cortex demonstrates that expressing ARHGAPI1B in bRGs in a primate substrate has the capacity to contribute to neocortical expansion and supragranular complexification during human evolution. ARGHAP11B-induced expansion of the cortical progenitor pool is mediated by metabolic changes in mitochondria, particularly increased glutaminolysis, a characteristic of highly mitotically active cells (10). This illustrates how cell metabolism-one of the most ancient of biological networks-participates in shaping the human lineage.

How does the increased rate of supragranular neuron production, resulting from $A R H G A P 11 B$ expression in OSVZ progenitors, affect the functional architecture of the cortex? And do these effects provide evolutionary insights? In the cortical hierarchy, areas are linked by a dense network of ascending (or bottom-up) and descending (or top-down) pathways forming a highly distributed hierarchy (11). Current theories of hierarchical processing of information in the cortex, including predictive coding theory, postulate 
that top-down messages signaling expectations interact in the supragranular layers with bottom-up activity from the sensory periphery, thereby enabling the brain to actively infer the causes of sensory stimulus (12). Recent structural analysis reveals that in the supragranular layers top-down and bottom up connections form two opposing streams thereby constituting a counterstream architecture (11) (see the figure).

During phylogeny there is a marked increase in the numbers of cortical areas, so that larger numbers of human supragranular neurons are required to integrate cortico-cortical circuits compared to non-human primates. In addition, because of the specific coding properties of the supragranular layers, increased in the numbers of supragranular neurons are expected to increase the circuit efficiency of these layers (13), which along with their complexification (14) could drive gains in computational power and the capacity to integrate top-down and bottom-up signals. The architecture of the primate brain has therefore evolved for the computational mechanisms that affect human perception and sense of self; this also has implications for the evolution of memory and learning.

The findings of Heide et al. illuminate how a molecular mechanism driving cortical development can scale up phylogenetically ancestral primate brains to the complexity of the human brain. ARHGAP11Bmediated humanization of the marmoset fetal cortex demonstrates the involvement of a human specific duplicated gene in the expansion of the supragranular layers. The effect of $A R H G A P 11 B$ expression on OSVZ progenitors and their cortical progeny, reinforces the importance of recent findings showing that human-specific regulatory elements are enriched in the OSVZ and the adult supragranular layers (15). Together, with the role of SRGAP2 (Slit-Robo-GTPase activating protein 2)- a human-specific duplicated gene that acts on cortical neuron complexity and synaptic circuitry (2), these findings point to crucial evolutionary adaptations converging on a cardinal feature of the cognitive architecture of the human cortex that underlie its unrivalled computational and cognitive performance. Future studies will need to address the effect of $A R H G A P 11 B$ expression at different time points in corticogenesis, its potential role in determining human specific cell types in the brain, and its intersection with the etiology of neurological disorders $(4,14$, $15)$.

\section{REFERENCES AND NOTES}

1.M. Y. Dennis, E. E. Eichler, Curr. Opin. Genet. Dev. 41, 44 (2016).

2.C. Charrier et al., Cell 149, 923 (2012).

3.M. Heide et al., Science 369, XXX (2020)

4.A. M. M. Sousa, K. A. Meyer, G. Santpere, F. O. Gulden, N. Sestan, Cell 170, 226 (2017).

5.I. H. Smart, C. Dehay, P. Giroud, M. Berland, H. Kennedy, Cereb. Cortex 12, 37 (2002).

6.D. V. Hansen, J. H. Lui, P. R. Parker, A. R. Kriegstein. Nature 464, 554 (2010).

7.M. Betizeau et al., Neuron 80, 442 (2013).

8.M. Florio et al., Science 347, 1465 (2015).

9.N. Kalebic et al., Elife 7, e41241 (2018).

10.T. Namba et al., Neuron 105, 867 (2020).

11.J. Vezoli et al., bioRxiv, 2020.2004.2008.032706 (2020).

12.R. P. Rao, D. H. Ballard, Nat. Neurosci. 2, 79 (1999).

13.K. D. Harris, T. D. Mrsic-Flogel, Nature 503, 51 (2013).

14. J. Berg et al., bioRxiv, 2020.2003.2031.018820 (2020).

15.H. Won, J. Huang, C. K. Opland, C. L. Hartl, D. H. Geschwind. Nat. Commun. 10, 2396 (2019). 


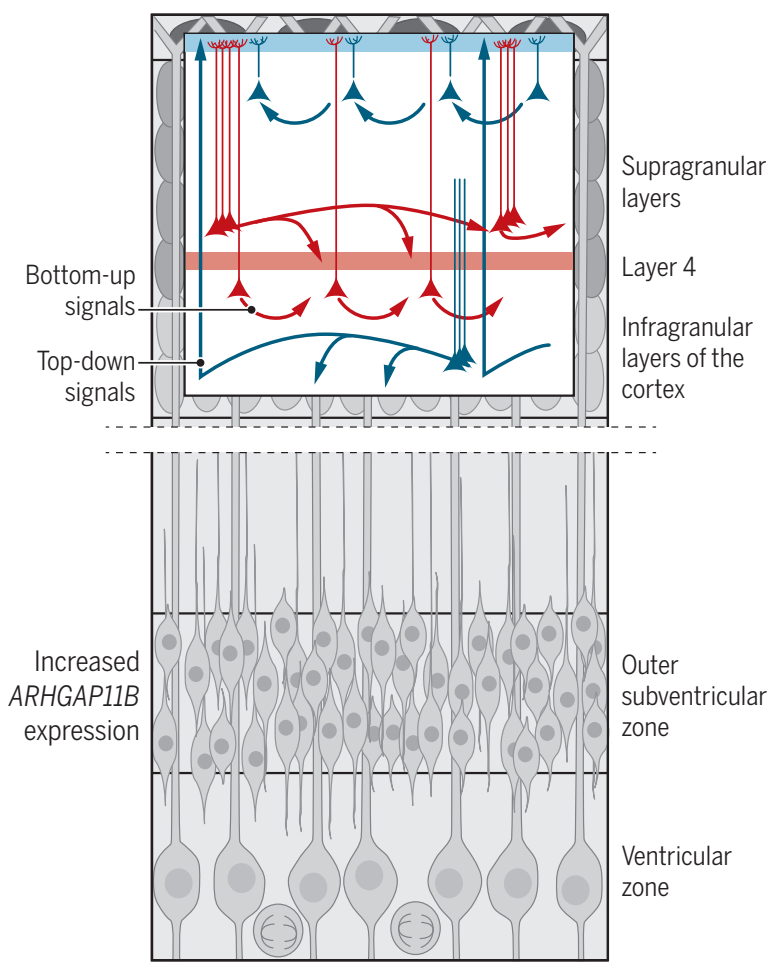

\section{Shaping the human cortex}

Heide et al. show that ARHGAP11B [Rho guanosine triphosphatase (GTPase) activating protein 11B] boosts proliferation in the outer subventricular zone, leading to increased production of cells destined for the supragranular layers. The counterstream architecture of the supragranular layers comprises feedback projections carrying top-down signals (blue arrows) that interact with feedforward projections (red arrows) carrying bottom-up sensory signals. The integration of these two pathways into the local microcircuit is a key feature of hierarchical processing in the primate cortex and will be favored by increased numbers of supragranular neurons. 